\title{
CONHECIMENTO SOBRE BIOSSEGURANÇA ENTRE MANICURES: necessidade de educação em saúde
}

\author{
KNOWLEDGE ABOUT BIOSECURITY AMONG MANICURES: \\ need of health education
}

Andressa Fernanda Silva', Cristiane Aparecida Silveira²

\section{RESUMO}

Objetivo: Identificar os conhecimentos sobre biossegurança de manicures, caracterizando-as quanto aos seus dados sociodemográficas e laborais. Metodologia: Trata-se de um estudo descritivo, epidemiológico, realizado em um município de Minas Gerais, através de entrevista semiestruturada. Os dados foram analisados a partir da estatística descritiva. Resultado: Do total, 72 (98,63\%) eram mulheres; idade média de 33,81 anos, a maioria tinha renda familiar de até dois salários mínimos; 64 $(87,67 \%)$ não tinham registro em carteira. Quanto ao conhecimento sobre esterilização, $26(36,63 \%)$ lavavam os materiais antes, $47(64,38 \%)$ utilizavam a estufa, $14(19,18 \%)$ não realizavam nenhum procedimento e somente $46(63,01 \%)$ usavam luvas. Do total, $56(22,67 \%)$ acreditavam que poderiam desenvolver LER/DORT. Ressalta-se que 47 (64,38\%) fizeram cursos profissionalizantes e $65(89,04 \%)$ receberam orientações sobre a correta forma de limpeza, desinfecção e esterilização. Conclusão: São necessárias políticas públicas enfocando à saúde do trabalhador, além de ações de educação em saúde voltadas a esta categoria.

Descritores: Saúde do Trabalhador; Enfermagem; Centros de Embelezamento e Estética; Saúde das Mulheres.

\section{ABSTRACT}

Objective: To identify the knowledge of manicure biosecurity, characterizing them as to their sociodemographic and labor data. Methodology: This is a descriptive, epidemiological study conducted in a city in Minas Gerais, through semi-structured interviews. Data were analyzed using descriptive statistics. Results: A total of $72(98.63 \%)$ were women; average age of 33.81 years, most had family income of up to two minimum wages; $64(87.67 \%)$ had no unregistered. Regarding knowledge about sterilization, $26(36.63 \%)$ washed their materials before, 47 (64.38\%) used the greenhouse, $14(19.18 \%)$ did not perform any procedure and only $46(63.01 \%)$ used gloves. Of the total, $56(22.67 \%)$ believed they could develop RSI / MSDs. It is noteworthy that $47(64.38 \%)$ were professional and 65 courses $(89.04 \%)$ received guidance on the correct way of cleaning, disinfection and sterilization. Conclusion: focusing on public policies are needed to worker health, and health education actions in this category.

Descriptors: Health Workers; Nursing; Beauty and Esthetics Centers; Women's Health
${ }^{1}$ Graduada em Enfermagem pela Ponficia Universidade Catolica de Minas Gerais (PUC MG), Poços de Caldas, MG, Brasil.

2 Doutora em Enfermagem pela Escola de Enfermagem de Ribeirão Preto da USP (EERPUSP), Ribeirão Preto, SP, Brasil. 


\section{Introdução}

As condições de saúde e doença estão ligadas às más condições de vida, de trabalho, de alimentação, à baixa qualificação profissional, que levam os indivíduos a perpetuação da pobreza ${ }^{1}$. As condições de trabalho desfavoráveis além de exporem os trabalhadores a vários riscos, culminam com o adoecimento e grande ônus aos serviços de saúde².

Tanto nos empregos formais quanto aos informais existem ambientes laborais inapropriados ${ }^{3}$. Entretanto, a informalidade constitui-se uma dificuldade extra na percepção dos riscos para o trabalhador ${ }^{4}$, isto porque a falta do empregador e da fiscalização faz com que muitas vezes o profissional não tenha proteção adequada e/ou não siga as orientações de prevenção.

Uma das profissões que trabalham na informalidade é a manicure que, como outras tipicamente femininas, são vistas com subalternidade e discriminação. Caracterizadas como trabalhadoras fragilizadas, vivenciando condições laborais deletérias e situação de informalidade e inseguras devido sua situação previdenciária e instabilidade financeira. São trabalhadoras que passam despercebidos perante a sociedade e as políticas de saúde ao trabalhador ${ }^{5}$.

Quanto ao risco ocupacional, as decisões e práticas de submeter-se ao mesmo estão constantemente presentes no seu cotidiano. O risco é entendido como a possibilidade diferenciada de certas exposições e/ou experiências causarem danos à integridade física, tais como doenças, desgaste, sofrimento, perda, morte, entre outros ${ }^{6}$.

As atividades laborais desses profissionais caracterizadas por longas jornadas, posições desconfortáveis, execução de movimentos repetitivos, contato com sangue e possibilidade de contágio de doenças como HIVIAIDS, Hepatite B ou outras. Situação agravada pela falta de percepção dos riscos que suas atividades as expõem e pelo fato de os trabalhadores não associarem o trabalho informal a maior risco de adoecimento ou surgimento de doenças ${ }^{7}$, seja pelo processo de desinfecção e esterilização que utilizam, não serem eficazes ou serem realizados de forma inapropriada ou não convencionais ${ }^{8}$.

Os trabalhadores necessitam de orientações sobre os riscos que suas atividades laborais os expõem. $O$ acesso a informações como o uso de Equipamentos de Proteção Individual (EPI) e a percepção dos riscos, podem resultar em estratégias que amenizem a exposição aos mesmos e até mesmo redução dos números de acidentes de trabalho ${ }^{9}$.

Entretanto essa adesão aos EPI por profissionais do segmento da beleza e estética depende, todavia, do conhecimento dos envolvidos (empregadores, empregados e clientes) acerca da biossegurança ${ }^{10}$.

Diante do exposto, o objetivo do presente estudo foi identificar os conhecimentos sobre biossegurança de manicures, caracterizando-as quanto aos seus dados sociodemográficas e laborais.

\section{Metodologia}

Trata-se de um estudo descritivo, analítico com abordagem quantitativa de caráter epidemiológico, realizado em um município do interior de Minas Gerais com população estimada de 41.368 habitantes.

Os profissionais foram entrevistados nos salões de beleza e em suas casas. Para seleção dos salões, buscaram-se inicialmente aqueles com alvará de funcionamento emitido pela Vigilância. Como essa não tinha um controle atualizado, optou-se por mapear por bairros os salões de beleza encontrados, independentemente do alvará da prefeitura, e as entrevistas foram realizadas no sentido de abranger todos os estabelecimentos. Durante as entrevistas as próprias manicures indicavam outros salões que eram, então, visitados.

Como critério de inclusão utilizou-se: manicure/pedicure quando presente nos salões de beleza, independente de ser proprietário ou empregado, independente de sexo, idade mínima de 18 anos e com experiência profissional mínima de um ano no segmento. Como critério de exclusão definiu-se menor de 18 anos de idade, com menos de seis messes de experiência na profissão. Após, constituíram-se participantes do estudo 73 trabalhadores $(n=73)$.

A coleta de dados ocorreu entre os meses de Junho a Agosto de 2014. Foram entrevistados 73 indivíduos, os trabalhadores responderam ao questionário após assinatura de Termo de Consentimento Livre e Esclarecido.

Para a caracterização da amostra, utilizou-se o Instrumento de Coleta de Dados composto por perguntas abertas e fechadas, abordando informações demográficas (faixa etária, renda familiar e escolaridade) laborais (realização das praticas de esterilização, acidentes de trabalho) e situação de saúde (doenças que acreditam que possam adquirir devido as suas praticas profissionais).

Foram incluídos no presente estudo trabalhadores que exerçam os serviços de embelezamento e higiene e de tecnólogos e técnicos em terapias complementares e estéticas. 
Para a análise dos dados, construiu-se um banco de dados no programa Excel for Windows 2002, com dupla alimentação dos dados e foi realizada a análise estatística, utilizando o Statistical Package for the Social Sciences (SPSS) versão 17.0 .

Utilizou-se estatística descritiva para a caracterização da população estudada, realizada por meio do cálculo das frequências absolutas e percentuais das variáveis sociodemográficas, procedimentos de limpeza, desinfecção e esterilização e das orientações de saúde.

A pesquisa foi aprovada pelo Comitê de Ética em Pesquisa CAAE (22580913.9.0000.5137 em 07/04/2014) de acordo com as diretrizes da Resolução 466/12 do Conselho Nacional de Saúde ${ }^{11 .}$

\section{Resultados}

Foram estudados 73 trabalhadores que atuavam em serviços de embelezamento e de terapias complementares e estéticas. Observou-se predominância feminina 72 (98,63\%) dos entrevistados eram mulheres.

Tabela 1 - Distribuição de trabalhadores dos serviços de embelezamento e higiene e de tecnólogos e técnicos em terapias complementares e estéticas em relação aos dados sociodemográficos.

Minas Gerais, 2014. $(n=73)$

\begin{tabular}{|c|c|c|c|}
\hline \multicolumn{2}{|r|}{ Variáveis } & \multicolumn{2}{|c|}{ Total } \\
\hline \multirow{6}{*}{ Faixa etária } & 18 a 27 anos & 25 & 30,72 \\
\hline & 28 a 37 anos & 21 & 27,45 \\
\hline & 38 a 47 anos & 20 & 16,34 \\
\hline & 48 a 57 anos & 4 & 20,26 \\
\hline & 58 a 67 anos & 3 & 3,27 \\
\hline & Total & 73 & 100 \\
\hline \multirow{7}{*}{ Escolaridade } & EF Incompleto & 12 & 16,44 \\
\hline & EF Completo & 18 & 24,66 \\
\hline & EM Incompleto & 12 & 16,44 \\
\hline & EM Completo & 18 & 24,66 \\
\hline & ES Incompleto & 9 & 12,33 \\
\hline & ES Completo & 4 & 5,48 \\
\hline & Total & 73 & 100 \\
\hline \multirow{4}{*}{ Renda familiar } & 1 a 2 SM & 49 & 67,12 \\
\hline & 3 a 4 SM & 3 & 4,11 \\
\hline & 5 a 6 SM & 21 & 28,77 \\
\hline & Total & 73 & 100 \\
\hline \multirow{4}{*}{$\begin{array}{c}\text { Situação } \\
\text { Previdenciária }\end{array}$} & Empregado (Celetista) & 9 & 12,33 \\
\hline & Contribuintes individuais (autônomas) & 21 & 28,77 \\
\hline & Não seguradas & 43 & 58,9 \\
\hline & Total & 73 & 100 \\
\hline
\end{tabular}

Legenda: f: frequencia; EF: Ensino Fundamental; EM: Ensino Médio; ES: Ensino Superior Fonte: Dados da pesquisa 
Em relação à faixa etária, $25(30,72 \%)$ tinham de 18 a 27 anos e a idade média foi de 33,81 anos (mediana: 35 e desvio padrão 10,439). Analisando a escolaridade, 30 (41,10 \%) não tinham nem o ensino médio e 4 (5,48\%) possuíam o ensino superior completo.

A renda familiar dos entrevistados, em sua maioria, correspondia em até dois salários mínimos $49(67,12 \%)$ e de 1 a 7 pessoas viviam com essa renda (média: 2,11; mediana: 2 e desvio padrão de 1,035).

Do total 64 (87,67\%) não tinham registro em carteira; dessas 21 (28,77\%) eram contribuintes individuais (autônoma) sendo seguradas pelo Instituto Nacional de Seguro Social (INSS). Entretanto, 43 eram trabalhadores informais, ou seja, não contribuem com a Previdência Social e 9 (12,33\%) empregadas (celetistas).

Tabela 2 - Distribuição de trabalhadores dos serviços de embelezamento e higiene e de tecnólogos e técnicos em terapias complementares e estéticas entrevistados em relação aos procedimentos de limpeza, desinfecção e esterilização. Minas Gerais, 2014.(n=73)

\begin{tabular}{|c|c|c|c|}
\hline \multicolumn{2}{|r|}{ Variáveis } & \multicolumn{2}{|c|}{ Total } \\
\hline \multirow{6}{*}{$\begin{array}{c}\text { Realiza algum } \\
\text { procedimento } \\
\text { antes } \\
\text { esterilização }\end{array}$} & Lavagem (mecânica) & 26 & 36,63 \\
\hline & Passar álcool & 19 & 26,03 \\
\hline & Passar água sanitária & 2 & 2,74 \\
\hline & Passar glutaraldeído & 1 & 1,37 \\
\hline & $\begin{array}{l}\text { Não realiza nenhum } \\
\text { procedimento }\end{array}$ & 24 & 32,88 \\
\hline & Total & 73 & 100,00 \\
\hline \multirow{5}{*}{$\begin{array}{c}\text { Prática do } \\
\text { procedimentos } \\
\text { de desinfecção/ } \\
\text { esterilização }\end{array}$} & Estufa & 47 & 64,38 \\
\hline & Hipoclorito & 8 & 10,96 \\
\hline & $\begin{array}{c}\text { Não realiza nenhum } \\
\text { procedimento }\end{array}$ & 14 & 19,18 \\
\hline & Ambos & 4 & 5,48 \\
\hline & Total & 73 & 100,00 \\
\hline \multirow{9}{*}{$\begin{array}{c}\text { Doenças que } \\
\text { você acredita } \\
\text { que pode } \\
\text { adquirir/ } \\
\text { desenvolver com } \\
\text { o seu trabalho * }\end{array}$} & LER/DORT & 56 & 22,67 \\
\hline & Doenças ósseas & 42 & 17,00 \\
\hline & Hepatites & 49 & 19,84 \\
\hline & Micoses & 48 & 19,43 \\
\hline & AIDS & 39 & 15,79 \\
\hline & Tuberculose & 8 & 3,24 \\
\hline & Outras & 3 & 1,21 \\
\hline & Nenhuma & 2 & 0,81 \\
\hline & Total & 247 & 100,00 \\
\hline
\end{tabular}

*Total diferentes do $\mathrm{n}$ pois os sujeitos podiam marcar mais de uma alternativa.

Legenda: f: frequência;

Fonte: Dados da pesquisa

Antes da esterilização, 26 (36,63\%) preparavam o material a ser esterilizado realizando lavagem (mecânica) e 19(26,03\%) passavam álcool nos utensílios. 
Quanto à desinfecção/esterilização, 47 (64,38\%) utilizavam a estufa, os chamados "forninhos", porém não souberam informar a temperatura utilizada no procedimento e $14(19,18 \%)$ não realizavam nenhum procedimento de desinfecção ou esterilização nos materiais do salão.

Quando questionados sobre doenças que possam desenvolver pelo trabalho $56(22,67 \%)$ Lesão por Esforço Repetitivo/ Doenças Osteomusculares Relacionadas ao Trabalho (LER/DORT) e 42 (17\%) de forma genérica doenças ósseas. Indagados sobre o uso dos EPI, 46(63,01\%) usavam luvas , 19 (26,03\%) usavam aventais, $7(9,59 \%)$ de máscaras e $6(8,22 \%)$ óculos.

Ressalta-se que $47(64,38 \%)$ afirmaram terem feito cursos profissionalizantes e $44(60,27 \%)$ relataram que nos cursos receberam orientações sobre sua própria saúde e formas de prevenção de doenças relacionados ao trabalho e 65 $(89,04 \%)$ receberam orientações sobre a correta forma de limpeza, desinfecção e esterilização dos materiais e instrumentos. Entretanto, ao analisar as respostas sobre como são tratados esses processos, percebe-se um grande desconhecimento destes temas e situações de grande risco a saúde destas trabalhadoras e de suas clientes como exposto na Tabela 3.

Tabela 3 - Distribuição de trabalhadores dos serviços de embelezamento e higiene e de tecnólogos e técnicos em terapias complementares e estéticas entrevistados em relação a orientações de saúde. Minas Gerais, 2014 ( $n=73)$.

\begin{tabular}{|c|c|c|c|c|c|}
\hline \multirow{2}{*}{\multicolumn{2}{|c|}{ Variáveis }} & \multicolumn{2}{|c|}{ Postura } & \multicolumn{2}{|c|}{ Doenças no trabalho } \\
\hline & & $f$ & $\%$ & $\mathbf{f}$ & $\%$ \\
\hline \multirow{8}{*}{$\begin{array}{l}\text { Fonte das } \\
\text { orientações }\end{array}$} & Profissionais de Saúde & 21 & 28,77 & 15 & 20,55 \\
\hline & Professor & 15 & 20,55 & 27 & 36,99 \\
\hline & Usuários dos serviços & 3 & 4,11 & 5 & 6,85 \\
\hline & Educador físico & 2 & 2,74 & - & - \\
\hline & TV/ internet & 1 & 1,37 & 7 & 9,59 \\
\hline & Outros & 4 & 5,48 & 10 & 13,70 \\
\hline & $\begin{array}{c}\text { Não receberam } \\
\text { orientações }\end{array}$ & 27 & 36,99 & 9 & 12,33 \\
\hline & Total & 73 & 100,00 & 73 & 100,00 \\
\hline
\end{tabular}

Em relação às orientações de saúde, $64(87,67 \%)$ relataram ter recebido as orientações sobre doenças no trabalho e $46(33,58 \%)$ sobre postura.

Observou-se que $27(50,00 \%)$ dos trabalhadores receberam as orientações sobre as doenças infectocontagiosas e $15(21,43 \%)$ sobre postura nos curso de capacitação de trabalhadores dos serviços de embelezamento e higiene e de tecnólogos e técnicos em terapias complementares e estéticas com seus professores.

Os profissionais de saúde foram responsáveis pela orientação de $21(30,00 \%)$ manicures sobre postura e 15 $(17,46 \%)$ sobre doenças do trabalho.

\section{Discussão}

O perfil da população estudada mostra diversas características ligadas as fragilidades sociais e laborais: maioria mulher, baixa escolaridade, baixa renda e sem proteção previdenciária/trabalhista (informais).

As mulheres ainda enfrentam situações de desigualdade relacionadas ao gênero nos aspectos sociais e laborais: vivenciam a subalternidade por meio de trabalhos informais, baixas remunerações, sem acesso a educação e informações, pouca participação na tomada de decisões. Situação agravada pelo acumulo de tarefas e responsabilidades domésticas ${ }^{12}$.

Apesar da inserção feminina no mercado de trabalho em países desenvolvidos nos últimos anos ter crescido, as mulheres ainda continuam como as grandes responsáveis pelas tarefas em seus lares. Tais atividades consomem energia e tempo, o que pode estagnar sua ascensão profissional ${ }^{13}$, expô-las a adoecimento pela sobrecarga de trabalho ${ }^{12}$, tornálas mais vulneráveis a danos físicos e mentais ${ }^{14}$, e à diminuição salarial quando comparada aos homens ${ }^{15}$. 
Os níveis de escolaridade estão diretamente relacionados com a compreensão de informações relacionadas à saúde, facilitando o relacionamento entre profissional de saúde como agente educador e paciente ${ }^{16}$.

A literatura mostra que também a renda pode impactar de forma negativa o estado de saúde, tornando-os mais predispostos a ocorrência de patologias ${ }^{17}$, bem como a idade, fator importante a ser considerado, pois predispõe às mulheres à diversas patologias, especialmente quando já se tem mais de cinco anos de atuação profissional ${ }^{18}$.

Além destes fatores, as próprias condições de trabalho têm causado o adoecimento e o incremento do número de Acidentes de Trabalho (AT). Pode-se constatar essa realidade pela alta incidência de Lesão por Esforço Repetitivo/ Doença Osteomuscular Relacionada ao Trabalho, absenteísmo, acidentes com perfurocortantes, entre outros ${ }^{19}$.

Os índices de AT nessa categoria são subestimados devido à alta subnotificação ${ }^{10}$. Desconhecendo a epidemiologia dessas moléstias, estabelecer meios de prevenção se torna difícil ${ }^{20}$.

Os AT são fenômenos complexos e impactantes na sociedade, pelo caráter financeiro e pelos agravos à saúde dos profissionais. A carência de conhecimentos sobre a ocorrência de AT e os procedimentos a serem realizados pós-acidente tornam a temática complexa, dificultando a prevenção dos agravos. Apesar de bem exposto na literatura e na legislação, os procedimentos a serem tomados pós acidente ocupacional por perfurocortantes é desconhecido por esses profissionais são ${ }^{21}$.

Além disso, a realização das práticas corretas de higienização e esterilização aliadas as demais estratégias de biossegurança ${ }^{5}$. A quebra dessas práticas consistem a uma quebra nos processos de biossegurança que pode resultar em contaminação dos usuários dos materiais, além de transmissão de doenças ${ }^{18}$.

A educação em saúde pode ser uma estratégia profilática e no enfrentamento ao adoecimento. 0 processo pedagógico consiste em ofertar conhecimentos sobre saúde à população com o intuito de mudanças de hábitos e adesão às práticas mais saudáveis evitando 0 adoecimento e a exposição a riscos ${ }^{16}$.

Os procedimentos adequados se iniciam com a lavagem dos materiais como alicates, palitos de metal, espátulas, entre outros, com água e sabão líquido. Nesta etapa o profissional deve fazer uso de luvas de borracha e uma escova de cerdas macias. Em seguida, os materiais devem ser enxaguados, secos com papel toalha e armazenados em embalagens adequadas para esterilização em autoclaves ou estufas. Na autoclave é feita por meio de vapor sob pressão, com tempo e temperatura variando conforme fabricantes. Nas estufas de calor seco, os materiais devem permanecer durante uma hora a $170^{\circ} \mathrm{C}$ ou duas horas a $160^{\circ} \mathrm{C}$. A estufa deve possuir um termostato e termômetro externo ${ }^{22}$.

O EPI mais utilizado foram as luvas, dado este condizente com a literatura, mas não identificado durante o trabalho de campo ${ }^{21}$.

A percepção do trabalhador em relação aos riscos ocupacionais aos quais são expostos, pode influenciar no uso dos EPI e a ocorrência de AT, tornando as condições laborais menos deletérias à sua saúde ${ }^{23}$.

Um dos fatores que podem interferir na adoção das medidas de biossegurança, é falta de conhecimento e uma justificativa frequente para a não adesão aos EPI é o incômodo ou desconforto e a alergia durante 0 uso ${ }^{18}$.

A maioria dos cursos profissionalizantes tem seus projetos pedagógicos focados no seguimento estético e embelezamento, não enfatizando a biossegurança e o autocuidado do trabalhador. Esta lacuna pode ser uma das causas do desconhecimento dos riscos que os profissionais e clientes são expostos ${ }^{8}$.

A proposta original da Lei 12.592/2012 que regula as atividades profissionais de manicures, barbeiros e outras atividades dos segmentos de estética e beleza no Brasil, estabelecia a exigência de ter concluído, no mínimo, o ensino fundamental e ter curso técnico ou treinamento profissional ou diploma comprovando a habilidade para atuação específica ou no caso de não serem portadores dos diplomas ou de certificados citados anteriormente, estarem exercendo a profissão há pelo menos 1 (um) ano, contado da data de publicação da Lei. Tais exigências foram vetadas com a justificativa que infringiam o disposto constitucional sobre o livre exercício de qualquer trabalho, ofício ou profissão, cabendo a imposição de restrições apenas quando houver a possibilidade de ocorrer algum dano à sociedade ${ }^{24}$. Entretanto, como exposto, a não capacitação oferece risco não só a saúde dos clientes, mas principalmente à sua própria saúde.

A percepção dos riscos nos profissionais que possuem cursos formais é mais positiva, aliando a uma melhor proteção. Caso houvesse a obrigatoriedade de formação dos profissionais em cursos regulares prevendo uma disciplina sobre biossegurança e as escolas propusessem atividades extracurriculares (palestras, visitas técnicas), haveria maiores chances de capacitação sobre este assunto ${ }^{18}$.

Além de uma boa formação técnica é imprescindível a atuação do setor saúde com o trabalho de uma equipe multidisciplinar em saúde é fundamental para prevenção de agravos no ambiente laboral ${ }^{25}$. 
As práticas de educação em saúde são um dos princípios norteadores da assistência em saúde e deve ser foco da vigilância em saúde do trabalhador, sendo medida preventiva dos adoecimentos relacionados ao trabalho. Educação sanitária que trabalhe a prevenção e o controle dos riscos ocupacionais consiste no fortalecimento das políticas de saúde do trabalhador, resultando em maior proteção trabalhista ${ }^{26}$. A ação educativa eficiente consiste em uma didática acessivel, abrangendo temas de interesses à saúde destes trabalhadores, seja através de plaestras, aulas ou cartilhas ${ }^{27}$.

Seguindo os princípios da Política Nacional de Saúde do Trabalhador e da Trabalhadora, os profissionais da atenção primária possuem a responsabilidade de desenvolver atividades e assistir os trabalhadores de sua área adjacente. A atenção primária corresponde ao primeiro estagio da estruturação da Rede Nacional de Atenção Integral à Saúde do Trabalhador (RENAST) no contexto da Rede de Atenção à Saúde. Assim sendo os profissionais da atenção primária devem, caracterizar os trabalhadores de sua cobertura, identificando os fatores nocivos à saúde, que sejam influenciáveis pelas condições laborais ${ }^{28}$.

\section{Considerações Finais}

O estudo identificou o desconhecimento sobre biossegurança entre manicures e similares. Trata-se de uma profissão geralmente informal, desprovida de proteção legal e que trabalha com diversos riscos ocupacionais, sobretudo biológicos e ergonômicos.

Devido a falta de uma disciplina obrigatória de biossegurança em seus cursos de formação/capacitação, muitas se expõem cotidianamente e também aos seus clientes. As lacunas de conhecimento encontradas no estudo pode ser um amplo campo para a atuação dos profissionais de saúde, na promoção e prevenção da saúde visando amenizar os futuros impactos na saúde.

A educação em saúde pode ser uma estratégia aliada às praticas de biossegurança para promover a educação sanitária e a vigilância à saúde do trabalhador. Ações mais enérgicas por parte das equipes interdisciplinares voltadas a vigilância em saúde do trabalhador com o objetivo de concretizar as mesmas.

São necessários investimentos para assistir à saúde de trabalhadores, em especial mulheres, carentes de assistência e a atenção à sua saúde. A busca pela redução da discriminação e desigualdade de gênero corresponde a um desafio global e devemos buscar e estimular condições laborais mais saudáveis a estas mulheres. Maiores inserções no mercado de trabalho, redução da informalidade, melhorar a qualidade de vida no trabalho, assegurar acesso a formação e educação, amenizar as diferenças relacionadas aos rendimentos correspondem aos grandes desafios para a igualdade de gênero e a nossa sociedade.

\section{Referências}

1. Grande AJ, Silva V, Manzatto L, Rocha TBX, Martins GC, Junior V, et al. Determinants of quality of life at workplace: clusterrandomized controlled trial. Rev Bras Med Esporte [Internet]. outubro de 2013 [citado 12 de março de 2015];19(5):371-5. Disponível em:: http://www.scielo.br/scielo.php?script=sci_abstract\&pid=S1517-86922013000500015\&lng=en\&nrm=iso\&tng=pt

2. Lenis Ballesteros V, Arango L, Lucía Y, Urrego C, Milena Y. Health and informal work conditions among recyclers in the rural area of Medellin, Colombia, 2008. Rev Saúde Pública [Internet]. outubro de 2012 [citado 12 de janeiro de 2015];46(5):866-74. Disponível em:: http://www.scielosp.org/scielo.php?script=sci_abstract\&pid=S0034-89102012000500014\&lng=en\&nrm=iso\&tlng=es

3. Saha A, Sadhu HG. Occupational injury proneness in young workers: a survey in stone quarries. J Occup Health. 2014;55(5):333-9.

4. Iriart JAB, Oliveira RP de, Xavier S da S, Costa AM da S, Araúijo GR de, Santana VS. Representations of informal jobs and health risks among housemaids and construction workers. Ciênc Saúde Coletiva. fevereiro de 2008;13(1):165-74.

5. Yoshida $\mathrm{CH}$, Oliveira RA de, Coelho PG, Fonseca FLA, Filipini R, Yoshida $\mathrm{CH}$, et al. Process of instrument sterilization in shops with manicure and pedicure services. Acta Paul Enferm [Internet]. fevereiro de 2014 [citado 26 de abril de 2015];27(1):18-22. Disponível em:: http://www.scielo.br/scielo.php?script=sci_abstract\&pid=S0103$21002014000100018 \&$ Ing=en\&nrm=iso\&tlng=en

6. Mendes R. Patologia do trabalho. 3a. Rio de Janeiro: Atheneu; 2013. 2070 p. 
7. Cortelli AFD. Procedimentos de biossegurança adotados por profissionais prestadores de serviços de manicure, pedicure, tatuagem, piercing e maquiagem definitiva no município de Jacaréi - SP [Internet] [Dissertação]. [São Paulo]: Universidade de São Paulo; 2012 [citado 19 de abril de 2016]. Disponível em:: http://www.teses.usp.br/teses/ disponiveis/6/6135/tde-26102012-114155/

8. Garbaccio JL, Oliveira AC. Biossegurança e risco ocupacional entre os profissionais do segmento de beleza e estética: revisão integrativa. Rev Eletrônica Enferm. 2012;14(3):702-11.

9. Gonçalves CG de O, Dias A. Three years of work-related accidents in a metallurgic plant: ways to its understanding. Ciênc Amp Saúde Coletiva [Internet]. fevereiro de 2011 [citado 28 de abril de 2015];16(2):635-46. Disponível em:: http:// www.scielo.br/scielo.php?script=sci_abstract\&pid=\$1413-81232011000200027\&lng=en\&nrm=iso\&tlng=pt

10. Garbaccio JL, Oliveira AC. Accidents with exposure to biological stuff, vaccine coverage and procedures after accidents among manicure/pedicure professionals. J Nurs UFPE Line JNUOLDOI 10520501012007. 2013;7(6):4421-4429.

11. Brasil. Resolução CNS no. 466, de 12 de dezembro de 2012-Estabelece as diretrizes e normas brasileiras regulamentadoras de pesquisas envolvendo seres humanos [Internet]. 2012. Disponível em:: http://www.rbbioetica.com. br/submissao/index.php/RBB/article/view/79

12. Organização das Nações Unidas. Igualdade de Gênero e Raça no Trabalho: avanços e desafios [Internet]. Brasília: Organização Internacional do Trabalho; 2010 [citado 19 de abril de 2016] p. 216. Disponível em:: http://www.oitbrasil.org. br/sites/default/files/topic/gender/pub/igualdade_genero_262.pdf

13. Oliveira ERA de, Garcia ÁL, Gomes MJ, Bittar TO, Pereira AC. Gender and perceived quality of life: research with professors from the health area. Ciênc Saúde Coletiva. março de 2012;17(3):741-7.

14. Hanklang S, Kaewboonchoo O, Silpasuwan P, Mungarndee SS. Musculoskeletal disorders among Thai women in construction-related work. Asia-Pac J Public Health Asia-Pac Acad Consort Public Health. 2014;26(2):196-202.

15. Cabral JPC, Burginski VM. Desemprego e Informalidade na Argentina: uma análise das diretrizes e recomendações da OIT e da Cepal para geração de trabalho e renda à população juvenil. Textos Context Porto Alegre. 5 de dezembro de 2011;10(2):227-43.

16. Slomp Junior H, Feuerwerker LCM, Land MGP. Educação em saúde ou projeto terapêutico compartilhado? 0 cuidado extravasa a dimensão pedagógica. Ciênc Saúde Coletiva. fevereiro de 2015;20(2):537-46.

17. Borges CM, Campos ACV, Vargas AMD, Ferreira EF e, Borges CM, Campos ACV, et al. Adult tooth loss profile in accordance with social capital and demographic and socioeconomic characteristics. Ciênc Saúde Coletiva. junho de 2014;19(6):1849-58.

18. Garbaccio JL. Conhecimento e adesão às medidas de biossegurança entre manicures e pedicures [Internet] [Tese de Doutorado]. [Belo Horizonte]: Universidade Federal de Minas Gerais; 2013 [citado 12 de setembro de 2014]. Disponível em:: http://www.bibliotecadigital.ufmg.br/dspace/handle/1843/GCPA-9GFK5W?show=full

19. Ansoleaga E, Vezina M, Montano R, Ansoleaga E, Vezina M, Montano R. Depressive symptoms and work-related stress in Chilean workers: differential conditions for males and females. Cad Saúde Pública [Internet]. janeiro de 2014 [citado 24 de setembro de 2014];30(1):107-18. Disponível em:: http://www.scielosp.org/scielo.php?script=sci_abstract\&pid=S0102$311 X 2014000100107 \&$ lng=en\&nrm=iso\&tlng=es

20. Gusmão GS, Oliveira AC de, Gama CS. Acidente de trabalho com material biológico: análise da ocorrência e do registro. Cogitare Enferm [Internet]. setembro de 2013 [citado 19 de outubro de 2014];18(3):558-64. Disponível em:: http://www.revenf.bvs.br/scielo.php?script=sci_abstract\&pid=S1414-85362013000300021\&lng=pt\&nrm=iso\&tlng=pt

21. Vilela RA de G, Almeida IM de, Mendes RWB. From surveillance to work-related accident prevention: the contribution of the ergonomics of the activity. Ciênc Amp Saúde Coletiva [Internet]. outubro de 2012 [citado 18 de outubro de 2014];17(10):2817-30. Disponivel em:: http://www.scielo.br/scielo.php?script=sci_abstract\&pid=S1413$81232012001000029 \&$ Ing=en\&nrm=iso\&tlng=pt

22. Brasil D de DA e HV. Hepatites virais longe do salão de beleza | Departamento de DST, Aids e Hepatites Virais [Internet]. 2011 [citado 8 de dezembro de 2014]. Disponível em:: http://www.aids.gov.br/pagina/prevenir-salao-beleza 23. Souza-Borges FRF de, Ribeiro LA, de Oliveira LCM. Occupational exposures to body fluids and behaviors regarding their prevention and post-exposure among medical and nursing students at a brazilian public university. Rev Inst Med Trop São Paulo. 2014;56(2):157-63. 
24. Brasil. Lei no 12.592, de 18 de janeiro de 2012. Dispõe sobre o exercício das atividades profissionais de Cabeleireiro, Barbeiro, Esteticista, Manicure, Pedicure, Depilador e Maquiador. [Internet]. 12.592 jan 18, 2012. Disponível em:: http:/l www.planalto.gov.br/ccivil_03/_ato2011-2014/2012/lei//12592.htm

25. Heidemann ITSB, Wosny A de M, Boehs AE, Heidemann ITSB, Wosny A de M, Boehs AE. Health promotion in primary care: study based on the Paulo Freire method. Ciênc Amp Saúde Coletiva [Internet]. agosto de 2014 [citado 18 de outubro de 2014];19(8):3553-9. Disponível em:: http://www.scielosp.org/scielo.php?script=sci_abstract\&pid=S1413$81232014000803553 \&$ lng=en\&nrm=iso\&tlng=pt

26. Pena PGL, Gomez CM. Health of subsistence fishermen and challenges for Occupational Health Surveillance. Ciênc Saúde Coletiva [Internet]. dezembro de 2014 [citado 26 de abril de 2015];19(12):4689-98. Disponível em:: http://www. scielo.br/scielo.php?script=sci_arttext\&pid=S1413-81232014001204689\&lng=en\&nrm=iso\&tlng=en

27. Goldschmidt IL. O teatro de Augusto Boal ea educação profissional em saúde. Trab Educ Saúde [Internet]. 2012 [citado 26 de abril de 2015];10(1):61-69. Disponível em:: http://www.scielo.br/scielo.php?pid=S1981$77462012000100004 \&$ script=sci_abstract

28. Ministério da Saúde, Gabinete do Ministro. BRASIL [Internet]. 2012 [citado 28 de abril de 2015]. Disponível em:: http:/l bvsms.saude.gov.br/bvs/saudelegis/gm/2012/prt1823_23_08_2012.html

\section{Cristiane Aparecida Silveira}

Endereço para correspondência - Rua: Pe. Francis Cletus Cox, $n^{\circ} 1661$, Bairro: Jardim Country Club, CEP: 37701355, Poços de Caldas, MG, Brasil.

E-mail: casilve@yahoo.com.br

Lattes: http://lattes.cnpq.br/8840798305145545

Andressa Fernanda Silva - andressa.fernanda18@hotmail.com

Enviado em 10 de março de 2016. Aceito em 29 de junho de 2016. 